* This manuscript should be cited as:

Zabelina, D. L., \& Silvia, P. J. (in press). Percolating ideas: The effects of caffeine on creative thinking and problem solving. Consciousness and Cognition.

\title{
Percolating Ideas:
}

\section{The Effects of Caffeine on Creative Thinking and Problem Solving}

\author{
Darya L. Zabelina \\ University of Arkansas \\ 480 Campus Drive \\ Fayetteville, AR 72701 USA \\ Paul J. Silvia \\ University of North Carolina at Greensboro \\ Department of Psychology \\ P.O. Box 26170 \\ Greensboro, NC 27402 USA
}

Author Note

We appreciate Tiffani Ng's assistance with the CRA task.

Correspondence concerning this article should be addressed to Darya L. Zabelina, Department of Psychological Science, 480 Campus Drive, Fayetteville, AR, 72701. Phone: (479) 575-5813. E-mail: dlzabeli@uark.edu. 


\begin{abstract}
Caffeine is the most widely consumed psychotropic drug in the world, with numerous studies documenting the effects of caffeine on people's alertness, vigilance, mood, concentration, and attentional focus. The effects of caffeine on creative thinking, however, remain unknown. In a randomized placebo-controlled between-subject double-blind design the present study investigated the effect of moderate caffeine consumption on creative problem solving (i.e., convergent thinking) and creative idea generation (i.e., divergent thinking). We found that participants who consumed $200 \mathrm{mg}$ of caffeine (approximately one $12 \mathrm{oz}$ cup of coffee, $n=44$ ), compared to those in the placebo condition $(n=44)$, showed significantly enhanced problemsolving abilities. Caffeine had no significant effects on creative generation or on working memory. The effects remained after controlling for participants' caffeine expectancies, whether they believed they consumed caffeine or a placebo, or for changes in mood. Possible mechanisms and future directions are discussed.
\end{abstract}

Keywords: Caffeine; creativity; convergent thinking; divergent thinking; mood 


\section{Introduction}

Caffeine is the most widely consumed psychotropic drug in the world (Bastia \& Schwarzschild, 2003), with $85 \%$ of adults in the US consuming at least one caffeinated beverage daily (Mitchell, Knight, Hockenberry, Teplansky, \& Hartman, 2014). Consuming caffeine has many cognitive benefits (for review, Glade, 2010): In low to moderate doses, caffeine has been shown to increase people's alertness (Hewlett \& Smith, 2007), improve vigilance and motor performance (Fine, Kobrick, Lieberman, Marlowe, Riley, \& Tharion, 1994), enhance concentration and attentional focus (Anderson, Revelle, \& Lynch, 1989; Rao, Hu, \& Nobre, 2005), and elevate mood (Smith, Clark, \& Gallagher, 1999; Smith, Sutherland, \& Christopher, 2005).

Caffeine's role in creative aspects of cognition, such as idea generation and problem solving, has received surprisingly little attention. In Western cultures, caffeine is stereotypically associated with creative occupations and lifestyles, from writers and their coffee to programmers and their energy drinks, and there's more than a kernel of truth to these stereotypes (Perry, 1999; Weinberg \& Bealer, 2004). Given the literature on caffeine and cognition, however, it is not clear whether caffeine should indeed improve creative cognition. The aim of the present study was thus to examine the effect of caffeine on creative cognition, namely creative problem solving (i.e., convergent thinking) and creative idea generation (i.e., divergent thinking).

Some literature presents evidence that lower attentional levels, and namely reduced cognitive control - which is linked with lower prefrontal cortex activity and lower cortical excitability - may be beneficial for creativity (Chrysikou 2019; Thompson-Schill, Ramscar, \& Chrysikou, 2009). There is also evidence from frontal lobe patients (Reverberi, Taraldo, D’Agostini, \& Skrap, 2005) and brain stimulation studies (Luft, Zioga, Banissy, \& Bhattacharya, 
2017) suggesting that lower prefrontal activity can indeed be beneficial for some types of creative thinking. Creative idea generation, however, has been shown to involve enhanced cognitive control together with spontaneous thought, as reflected in dynamic coupling between frontoparietal and default mode brain networks (for review, Beaty, Benedek, Silvia, \& Schacter, 2016; Zabelina \& Andrews-Hanna, 2016). Because caffeine typically enhances concentration and attentional focus (Anderson et al., 1989; Rao et al., 2005), it may differentially affect creative problem solving versus creative generation of ideas.

The Compound Remote Associates (CRA) task - a standard measure of problem solving (i.e., convergent thinking; Bowden \& Jung-Beeman, 2003)—presents participants with three seemingly unrelated words and asks them to generate a fourth word that forms a common word or a common compound with the three words. This task thus requires "convergence" on one correct solution. Successful performance on the CRA task relates to better executive control (Lee, Huggins, \& Therriault, 2014) and focused attention (Ostafin \& Kassman, 2012), so we expected that people who consume a moderate dose of caffeine (vs. placebo) would solve more CRA problems.

Convergent thinking is often contrasted with divergent thinking (creative idea generation), where there is no correct answer but rather a large set of responses that are suitably apt, novel, or interesting. On a typical measure of divergent thinking (e.g., the alternative uses task), participants are asked to generate multiple creative uses for a common object, such as a brick. Responses are scored for the number of uses generated (fluency), as well as for the novelty of each response (originality). Divergent thinking tasks recruit many cognitive processes, including top-down processes related to executive control network and bottom-up, associative processes related to the default-mode network (Benedek \& Fink, 2019). These top-down and 
bottom-up processes interact in complex ways during idea generation (Beaty et al., 2016;

Zabelina \& Andrews-Hanna, 2016), so predictions for caffeine and divergent thinking are much less certain than for convergent thinking, although greater executive control should broadly improve creative idea generation. Divergent thinking may be domain-specific (Palmiero, Nakatani, Raver, Belardinelli, \& van Leeuwen, 2010), therefore we also examined the effect of caffeine on verbal and visual divergent thinking separately.

In addition to convergent and divergent thinking, we assessed working memory and mood, which both are linked, albeit in complex ways, with creative thinking and problem solving (Baas, De Dreu, \& Nijstad, 2008; Smeekens \& Kane, 2016; Wiley \& Jarosz, 2012). Finally, we sought to examine and control for caffeine expectancy effects. Participants come to caffeine experiments with lay theories about how caffeine enhances mood and cognition-lay theories that are largely supported by research. As a result, expectancy effects are pervasive (e.g., Chan \& Maglio, 2019; Dawkins, Shahzad, Ahmed, \& Edmonds, 2011) and must be accounted for via research design and assessment. We thus used a double-blind, placebo-controlled design and assessed beliefs about caffeine's effects (Huntley \& Juliano, 2012) as possible moderators.

\section{Methods}

\section{1 Participants}

Eighty-eight people (60 females, 28 males, $)$, mean age $=21.58(S D=3.33 ; 5$ unreported $)$, majority White (78\%), participated in the current study. Because there is no prior work on the effects of caffeine on creativity, a formal power analysis was not possible, thus we based our projected sample size off of previous work examining the effects of psychotropic drugs on creativity (e.g., Benedek, Panzierer, Jauk, \& Neubauer, 2017), and the available time and resources to conduct the project. Our plan was to run the experiment within one semester, or 
until the sample size reached 80 people, whichever came last. Participants were recruited via the university newspaper, flyers posted around campus, and on the local National Public Radio station. Participants were invited to participate if they were between 18-35 years of age, consumed between 1-2 cups of caffeinated beverages per day at least 5 days a week, did not smoke, were native English speakers, and took no psychiatric medications or painkillers on a regular basis. The majority of participants reported coffee as their main source of caffeine ( 81 coffee, 4 tea, 1 energy drinks, 2 soda). Twenty participants drank one cup, and 59 participants drank two cups, of caffeinated beverages per day. Twenty-two participants drank caffeinated beverages five days a week, 23 six days a week, and 42 seven days a week. The study was approved by the local Institutional Review Board, and all participants provided informed consent.

\subsection{Experimental Design and Procedure}

The study examined the effects of caffeine in a randomized placebo-controlled betweensubject double-blind design. Each participant was randomly assigned to the caffeine or control condition. The experimental sessions contained up to six participants at a time and lasted up to 1 hour and 30 minutes. Participants were paid $\$ 15$ for their time.

Participants were asked to abstain from any caffeinated or alcoholic beverages from $4 \mathrm{pm}$ on the day prior to the session. They were told that to verify compliance with the abstinence requirements, they would be asked to provide a saliva sample at the beginning of the session (saliva samples were not collected). Sessions took place between 9:00 AM and 3:00 PM.

Participants were told that the university was conducting a study examining the effect of different doses of caffeine on cognitive performance (participants were thus unaware of the placebo condition). Participants took either a $200 \mathrm{mg}$ caffeine capsule or an identical-looking 
placebo capsule. Capsules were produced by a local pharmacy specifically for the present study. Participants completed a baseline mood questionnaire (Mood Time 1), took the capsule, and completed various questionnaires for exactly 20 minutes to allow time for caffeine to enter the bloodstream. They then completed the divergent thinking task (ATTA), convergent thinking task (CRA), and a working memory task (Keep Track).

At the end of the experiment participants completed the final mood questionnaire (Mood Time 2), and reported to what extent they expect caffeine to affect them (Caffeine Expectancy Questionnaire). Finally, they were informed that they were randomly assigned to one of the two experimental conditions (caffeine and placebo), and were asked to indicate a confidence level on a sliding scale $(1$ = definitely caffeine, $100=$ definitely placebo $)$ regarding whether they believed they had taken the caffeine or the placebo capsule. All computer tasks were administered on a Windows PC.

\subsection{Measures}

2.3.1 Convergent thinking. The Compound Remote Associates (CRA; Bowden \& JungBeeman, 2003) test was used to assess convergent problem solving. Participants were presented with 72 CRAs divided into three blocks ( 24 problems per block). The order of the problems was pseudo-randomized. For each problem, three cue words were presented in the center of the screen, and participants were instructed to press the spacebar if they knew the solution. Once the spacebar was pressed, the cue words disappeared, and participants typed the solution. The problem remained on the screen for 15 seconds and disappeared after this time in the event that the spacebar was not pressed. If participants typed in the solution, they were asked to indicate if they reached the solution by insight (a subjective Aha! experience; $F$ button) or by analysis (a deliberate step-by-step solution; $J$ button). They were told: “An insight or Aha! moment often 
feels sudden and surprising, as if you just know the solution works with all 3 problem words. In addition, you might be unable to explain how you reached the solution if you solved by insight. Analysis feels more deliberate and you might be more likely to report the steps you took to get to the solution." Normative data for the CRAs are provided by Bowden and Jung-Beeman (2003). Due to computer error, CRA data failed to record for 17 people, thus CRA data are reported for $n$ $=71$.

2.3.2 Divergent thinking. The Abbreviated Torrance Test for Adults (ATTA; Goff \& Torrance, 2002) was used to assess divergent thinking. The ATTA assesses both verbal (one activity), and figural (two activities) divergent thinking (3 mins each). The verbal activity instructs participants to generate problems that would arise from being able to walk or fly without being in an airplane or a similar vehicle. Figural activities present incomplete figures, and participants are asked to generate drawings incorporating the figures. In all three activities participants are instructed to "be creative" (Nusbaum, Silvia, \& Beaty, 2014; Said-Metwaly, Fernández-Castilla, Kyndt, \& Van den Noortgate, 2019). Responses are scored for fluency (i.e., the number of generated responses and drawings), and originality (i.e., how novel or original responses are compared to a large pool of normative responses). Scores were summed across the three activities for the final fluency and originality scores. The total divergent thinking score was calculated as the average of $z$-scored fluency and $z$-scored originality. Test developers report good reliability for the ATTA $(\mathrm{KR}-21=.84$; Goff \& Torrance, 2002).

2.3.3 Working memory. The Keep Track task was used to assess working memory (adapted from Miyake, Friedman, Emerson, Witzki, Howerter, \& Wager, 2000). The task instructed participants to keep track of the last word to appear on the screen from two to five different categories (animals, colors, countries, distances, metals, and relatives). On each trial 
participants saw the categories that they were to keep track of on the bottom of the screen, with categories remaining on the screen for the duration of the trial. 15-25 words appeared one at a time and remained on the screen for 2 seconds each. At the end of each trial the categories disappeared, and participants saw a "??," at which point they wrote down on a sheet of paper the last word they remembered from each of the category for that trial. There were 16 total trials (4 blocks of two, three, four, and five categories) in addition to the 2 practice trials. The dependent measure was recall accuracy. Four participants completed the Keep Track task incorrectly, so Keep Track data are reported for $n=84$.

2.3.4 Caffeine Expectancy Questionnaire (CaffEQ). The CAffEQ (Huntley \& Juliano, 2012) is a 47-item scale that assesses people's beliefs about how caffeine affects withdrawal/dependence, energy/work enhancement, appetite suppression, social/mood enhancement, physical performance enhancement, anxiety/negative physical effects, and sleep disturbance. Participants were asked to rate each statement on a 6-item Likert-type scale ranging from 1 (very unlikely) to 6 (very likely), indicating the extent to which they believed each consequence applied to them when they use caffeine. Examples of the items include: "Caffeine picks me up when I am feeling tired," and "Caffeine improves my attention." The total CaffEQ score is an average of all the items on the scale. The CaffEQ has excellent psychometric properties, including reliability $\alpha=.96$ (Huntley $\&$ Juliano, 2012). Two participants did not complete the CaffEQ, thus data are reported for $n=86$.

2.3.5 Mood. Mood was evaluated at the beginning (Time 1) and at the end (Time 2) of the session. Participants were asked to indicate on a sliding scale from 0 (not at all) to 100 (very) to what extent they were feeling happy, sad, excited, anxious, bored, and focused. Two 
participants did not complete the Time 1 questionnaire, and one participant did not complete the Time 2 questionnaire, thus $n=86$ for reported changes in mood.

\section{Results}

The caffeine and the placebo groups each consisted of 44 participants (16 male and 28 female participants in the caffeine group, and 12 male and 32 female participants in the placebo group). The two groups did not differ significantly in age, $t(82)=1.40, p=.165$, Cohen's $d=$ 31 .

\subsection{Effects of Caffeine on Convergent Problem Solving}

Independent-samples $t$-test indicated that participants who consumed caffeine solved significantly more CRA problems compared to the control group, $t(70)=2.37, p=.021$, Cohen's $d=.56$, suggesting a medium-size effect (Table 1; Figure 1). Importantly, analysis of covariance (ANCOVA) indicated that the effect remained significant after controlling for whether participants believed they took the caffeine or the placebo capsule, $F(1,69)=5.41, p=.023, \eta^{2}=$ .08. The effect was larger after controlling for individual differences in caffeine expectancy, $F(1,69)=8.81, p=.004, \eta^{2}=.12$. Further, the effect remained significant after controlling for the change in mood from Time 1 to Time 2: Happiness, $F(1,69)=5.22, p=.026, \eta^{2}=.07$; sadness, $F(1,69)=5.13, p=.027, \eta^{2}=.07$; excitement, $F(1,69)=5.55, p=.021, \eta^{2}=.08$; anxiety, $F(1,69)=5.70, p=.026, \eta^{2}=.09$; boredom, $F(1,69)=5.61, p=.021, \eta^{2}=.08$; and focus, $F(1,69)=5.23, p=.026, \eta^{2}=.07$; as well as after including all mood states as control variables, $F(1,61)=4.57, p=.037, \eta^{2}=.07$. Finally, the effect remained significant after controlling for gender, $F(1,69)=5.67, p=.020, \eta^{2}=.08$. 
Caffeine and placebo groups did not significantly differ in the CRA problems solved by insight or by analysis, $p \mathrm{~s}>.140$ (Table 1), indicating that caffeine improves convergent problem solving overall, regardless of the type of the solution.

Further, there were no statistically significant differences in error rates (false alarms) between the caffeine and the placebo conditions for CRA insight, CRA analysis, or CRA total solutions. Finally, participants in the caffeine (vs. placebo) condition were faster at solving CRA problems with insight, $p=.011$, while there were no statistically significant group differences in the speed of responses for CRA problems solved via analysis, nor for the total CRA reaction times (Table 1).

Table 1. The effect of caffeine $(n=34)$ and placebo $(n=37)$ on problem solving (convergent thinking) accuracy, errors (false alarms), and reaction times.

\begin{tabular}{|c|c|c|c|c|c|c|}
\hline & Caffeine & Place & & & & \\
\hline & Mean $(S D)$ & Mean $(S D)$ & $t$ & $p$ & Cohen's $d$ & $95 \% \mathrm{CI}$ \\
\hline CRA Total Acc & $29.09(7.30)$ & $25.00(7.24)$ & 2.37 & .021 & .56 & {$[.64 ; 7.53]$} \\
\hline CRA Insight Acc & $18.68(8.25)$ & $16.08(6.35)$ & 1.49 & .140 & .35 & {$[-.87 ; 6.07]$} \\
\hline CRA Analytic Acc & $10.41(8.64)$ & $8.92(6.24)$ & .84 & .405 & .20 & {$[-2.06 ; 5.05]$} \\
\hline CRA Total Err & $8.55(6.58)$ & $9.30(9.20)$ & .39 & .698 & .09 & {$[-4.61 ; 3.10]$} \\
\hline CRA Insight Err & $4.09(4.17)$ & $5.00(7.59)$ & .62 & .539 & .15 & {$[-3.86 ; 2.03]$} \\
\hline CRA Analytic Err & $4.12(4.46)$ & $4.41(3.61)$ & .27 & .790 & .07 & {$[-2.44 ; 1.86]$} \\
\hline CRA Total RT & $6.68(1.17)$ & $6.87(1.04)$ & .73 & .470 & .17 & {$[-.71 ; .33]$} \\
\hline CRA Insight RT & $5.73(1.29)$ & $6.56(1.38)$ & 2.60 & .011 & .62 & {$[-1.46 ;-.19]$} \\
\hline CRA Analytic RT & $8.61(1.89)$ & $7.95(2.08)$ & 1.38 & .174 & .33 & {$[-.30 ; 1.63]$} \\
\hline
\end{tabular}


Note. $\mathrm{CRA}=$ Compound Remote Associate $(\mathrm{CRA})$ test; Acc $=$ Accuracy $($ Number of correctly solved problems); Err $=$ Number of Errors; RT $=$ Reaction Time (in seconds). 95\% Confidence Intervals (CI) pertain to group differences.

\subsection{Effects of Caffeine on Divergent Thinking}

Overall, participants generated an average of $13.07(S D=3.57)$ responses, with an average originality score of $6.72(S D=3.42)$, consistent with the norms reported by the test developers (Goff \& Torrance, 2002). Descriptive statistics for the three separate activities of the ATTA are reported in Table 2.

Table 2. Descriptive statistics for the three activities of the Abbreviated Torrance Test for Adults.

\begin{tabular}{llll}
\hline & Mean $(S D)$ & Min & Max \\
& & & \\
\hline ATTA Activity 1 fluency (verbal) & $6.52(2.32)$ & 2 & 13 \\
ATTA Activity 2 fluency (figural) & $1.86(.41)$ & 1 & 3 \\
ATTA Activity 3 fluency (figural) & $4.67(2.35)$ & 0 & 9 \\
ATTA Activity 1 originality (verbal) & $2.82(1.76)$ & 0 & 7 \\
ATTA Activity 2 originality (figural) & $.95(.69)$ & 0 & 2 \\
ATTA Activity 3 originality (figural) & $2.94(2.48)$ & 0 & 9 \\
\hline
\end{tabular}
Note. ATTA $=$ Abbreviated Torrance Test for Adults.

Independent-samples $t$-test indicated that there were no significant differences in performance on the ATTA divergent thinking test (total divergent thinking, fluency, nor originality) between participants who consumed caffeine capsule and those in the control group, ps $>.406$ (Table 3), suggesting that caffeine did not influence divergent thinking. 
Table 3. The effect of caffeine $(n=44)$ and placebo $(n=44)$ on creative idea generation.

\begin{tabular}{lllllll}
\multicolumn{1}{l}{ Caffeine } & Placebo & & & & \\
\hline & Mean $(S D)$ & Mean $(S D)$ & $t$ & $p$ & Cohen's $d$ & $95 \%$ CI \\
& & & & & & \\
\hline ATTA Total & $.06(.67)$ & $-.06(.85)$ & .69 & .491 & .16 & {$[-.21 ; .43]$} \\
ATTA Fluency & $.10(.97)$ & $-.08(1.04)$ & .84 & .406 & .18 & {$[-.24 ; .61]$} \\
ATTA Originality & $.02(.90)$ & $-.03(1.11)$ & .22 & .829 & .05 & {$[-.38 ; .47]$} \\
ATTA Verbal & $.03(.88)$ & $-.06(.86)$ & .52 & .607 & .03 & {$[-.27 ; .47]$} \\
ATTA Figural & $.06(.59)$ & $-.03(.64)$ & .67 & .506 & .15 & {$[-.17 ; .35]$} \\
\hline
\end{tabular}

Note. ATTA $=$ Abbreviated Torrance Test for Adults. ATTA Total $=$ average of $z$-scored ATTA

Fluency and $z$-scored ATTA Originality. 95\% Confidence Intervals (CI) pertain to group differences.

We further examined the effect of caffeine on verbal and figural divergent thinking separately, by averaging the $z$-scored fluency and $z$-scored originality on Activity 1 (ATTA Verbal), and by averaging $z$-scored fluency and originality on Activities 2 and 3 (ATTA Figural). Results indicated that participants in the caffeine and the placebo conditions did not significantly differ in terms of their verbal $(p=.607)$ or figural $(p=.506)$ divergent thinking (Table 3$)$.

\subsection{Effect of Caffeine on Working Memory}

Independent-samples $t$-test indicated that there were no significant differences in performance on the Keep Track task between participants who consumed caffeine capsule and those in the control group, $p=.679$ (Table 4), indicating that caffeine had no influence on working memory. 
Table 4. The effect of caffeine $(n=43)$ and placebo $(n=41)$ on working memory and change in mood (before minus after) caffeine administration (caffeine $n=44$, placebo $n=42$ ).

\begin{tabular}{lllllll}
\multicolumn{1}{l}{ Caffeine } & Placebo & & & \\
\hline & Mean $(S D)$ & Mean $(S D)$ & $t$ & $p$ & Cohen's $d$ & $95 \%$ CI \\
& & & & & & \\
\hline & & & & & & \\
Keep Track & $41.37(6.60)$ & $40.78(6.43)$ & .42 & .679 & .09 & {$[-.21 ; .44]$} \\
Sadness & $-4.59(15.83)$ & $3.57(17.79)$ & 2.25 & .027 & .49 & {$[-15.38 ;-.95]$} \\
Happiness & $-3.59(15.89)$ & $-6.48(17.32)$ & .81 & .423 & .17 & {$[-4.24 ; 10.01]$} \\
Boredom & $-.27(31.06)$ & $6.14(30.34)$ & .97 & .336 & .21 & {$[-19.59 ; 6.76]$} \\
Anxiety & $-2.23(24.99)$ & $.51(25.14)$ & .48 & .633 & .11 & {$[-14.12 ; 8.65]$} \\
Focus & $8.23(24.29)$ & $5.81(22.34)$ & .48 & .633 & .10 & {$[-7.60 ; 12.44]$}
\end{tabular}

Note. ATTA $=$ Abbreviated Torrance Test for Adults. ATTA Total $=$ average of $z$-scored ATTA Fluency and $z$-scored ATTA Originality. 95\% Confidence Intervals (CI) pertain to group differences.

\subsection{Effects of Caffeine on Mood}

Independent samples $t$-test indicated that participants in the caffeine group reported decreased sadness from Time 1 to Time 2 (mean change $=-4.59, S D=15.83$ ), while participants in the placebo group reported increased sadness from Time 1 to Time 2 (mean change $=3.57, S D$ $=17.79), p=.027$. There were no significant differences between the two groups in reported change in mood between Time 1 and Time 2 for happiness, boredom, anxiety, or focus, $p \mathrm{~s}>.336$ (Table 4). 


\subsection{Effects of Caffeine Expectancy}

Independent samples $t$-test indicated that participants in the caffeine group were approximately at the level of chance for guessing whether they took the caffeine or the placebo capsule $(M=53.95, S D=31.34)$, while participants in the placebo group were more likely to correctly guess that they took the placebo capsule $(M=72.40, S D=23.38), t(85)=3.08, p=$ .003 , Cohen's $d=.67, \mathrm{CI}=[-30.35 ;-6.55]$.

Pearson correlations revealed that believing one consumed the caffeine capsule was significantly correlated with better performance on the working memory task, $r(86)=.28, p=$ .011 , but was not significantly correlated with any of the convergent, $p \mathrm{~s}>.15$ or divergent thinking variables $p \mathrm{~s}>.10$, nor with changes in mood, $p \mathrm{~s}>.09$ (Table 5). Furthermore, individual differences in caffeine expectancy significantly correlated with fewer CRA solutions by insight, $r(69)=-.29, p=.015$, but not with any other convergent, $p \mathrm{~s}>.20$ or divergent thinking variables, $p \mathrm{~s}>.31$, changes in mood, $p \mathrm{~s}>.11$ (Table 5), nor with performance on the Keep Track task, $r=-.07, p=.52$. 


\section{Table 5}

Pearson correlation coefficients for participants' beliefs whether they took the caffeine or the placebo capsule, caffeine expectancy (CaffEQ), problem solving (CRA), and creative thinking (ATTA) variables.

\begin{tabular}{|c|c|c|c|c|c|c|c|c|c|c|c|c|c|c|}
\hline 1 & 2 & 3 & 4 & 5 & 6 & 7 & 8 & 9 & 10 & 11 & 12 & 13 & 14 & 15 \\
\hline 1. Think Caffeine or Placebo -- & .01 & -.18 & -.11 & -.07 & -.13 & -.13 & -.06 & -.18 & -.01 & .17 & -.19 & .07 & -.06 & -.05 \\
\hline 2. Caffeine Expectancy & -- & .16 & $.29 *$ & -.19 & -.07 & -.06 & -.04 & -.11 & .007 & .09 & -.02 & .18 & .08 & .03 \\
\hline 2. CRA Total Acc & & -- & $.50 * *$ & $-.32 * *$ & $.33^{* *}$ & $.32 * *$ & .20 & $.33 * *$ & .18 & -.07 & .06 & -.01 & -.19 & .07 \\
\hline 3. CRA Insight Acc & & & -- & $-.38 * *$ & .07 & .08 & .03 & .02 & .10 & .00 & .13 & -.07 & -.20 & .04 \\
\hline 4. CRA Analytic Acc & & & & -- & -.07 & -.07 & -.04 & .04 & -.17 & -.02 & .09 & -.02 & .11 & -.08 \\
\hline 5. ATTA Total & & & & & -- & $.76^{* *}$ & $.76^{* *}$ & $.82 * *$ & $.74 * *$ & $-.21 \dagger$ & -.05 & .05 & .19 & .10 \\
\hline 6. ATTA Fluency & & & & & & -- & .16 & $.62 * *$ & $.58 * *$ & .00 & -.11 & .00 & .14 & .08 \\
\hline 7. ATTA Originality & & & & & & & -- & $.62 * *$ & $.55^{* *}$ & $-.31 * *$ & .03 & .07 & .15 & .08 \\
\hline 8. ATTA Verbal & & & & & & & & -- & $.22 *$ & -.18 & -.07 & .11 & .11 & .03 \\
\hline 9. ATTA Figural & & & & & & & & & -- & -.14 & -.01 & -.05 & .19 & .15 \\
\hline 10. Sadness & & & & & & & & & & -- & $-.38 * *$ & .11 & .06 & $-.26^{*}$ \\
\hline 11. Happiness & & & & & & & & & & & -- & $-.20 \dagger$ & -.01 & $.27^{*}$ \\
\hline 12. Boredom & & & & & & & & & & & & -- & -.10 & $-.37 * *$ \\
\hline 13. Anxiety & & & & & & & & & & & & & -- & .16 \\
\hline 14. Focus & & & & & & & & & & & & & & -- \\
\hline
\end{tabular}

Note. $\mathrm{CRA}=$ Compound Remote Associate (CRA) test; ATTA = Abbreviated Torrance Test for Adults; CaffEQ $=$ Caffeine Expectancy Questionnaire. $\uparrow p<.10$ $* p<.05 * * p<.01$. 


\section{Discussion}

The present study investigated the effect of caffeine on creative thinking and problemsolving abilities. In a double-blind placebo-controlled design study, we found that in people who consume a moderate dose of caffeine on a daily basis, consuming $200 \mathrm{mg}$ of caffeine (approximately equal to one $12 \mathrm{oz}$ cup of coffee) in a laboratory session significantly enhanced convergent problem-solving ability, but had no effect on divergent thinking or working memory. These effects remained when controlling for individual differences in caffeine expectancy, for believing whether or not one consumed a caffeine capsule, and for changes in mood throughout the session.

The effects of caffeine on creative problem-solving is consistent with the broader literature on caffeine and cognition. Research consistently shows enhanced concentration and attentional focus associated with caffeine consumption (Rao et al., 2005), and such attentional improvements are reasonable mechanisms for changes in convergent problem-solving tasks with correct answers. Further, caffeine's effect on higher-order cognitive processes, such as enhanced response inhibition (Barry, Johnstone, Clarke, Rushby, Brown, \& McKenzie, 2007), and enhanced performance on selective visual attention tasks (Brunyé, Mahoney, Lieberman, \& Taylor, 2010; Lorist, Snel, Kok, \& Mulder,1996) suggests that enhanced prefrontal activity may facilitate convergent problem solving. Caffeine did not differentially affect insight versus analytical solutions, indicating that caffeine improves convergent problem solving overall, regardless of the approach.

Although not predicted, consumption of $200 \mathrm{mg}$ of caffeine resulted in faster problemsolving by insight, which may reflect faster processing speed associated with caffeine 
consumption (Kamimori, McLellan, Tate, Voss, Niro, \& Lieberman, 2015). Deliberate, step-bystep analytical solutions were not affected by caffeine.

Notably, caffeine did not affect creative idea generation, measured with the ATTA battery of divergent thinking tasks (Goff \& Torrance, 2002). Based on previous literature showing that divergent thinking is linked with increased alpha activity - a common maker of relaxation and network inhibition (for review, Stevens \& Zabelina, 2019), and with caffeine resulting in global reduction in EEG power in the alpha activity (Barry, Rushby, Wallace, Clarke, Johnstone, \& Zlojutro, 2005), it is reasonable to expect caffeine leading to worse performance on divergent thinking tasks. Other line of work, however, reports complex interactions between top-down and bottom-up cognitive processes during divergent thinking (Beaty et al., 2016; Zabelina \& Andrews-Hanna, 2016), therefore it is not clear whether caffeine should indeed have any affect on divergent thinking. It is also possible that $200 \mathrm{mg}$ is not a sufficient dose to impact idea generation during divergent thinking, but higher amounts (e.g., 400 $\mathrm{mg}$ ) would result in reduced idea generation abilities.

Although the ATTA is widely used and is based on the venerable Torrance Tests of Creative Thinking (Torrance, 2008), its markers of originality are strongly affected by fluency (Silvia et al., 2008), often correlating close to .90 in large samples. Studying ways of separating quantity (fluency) and quality (creativity) has been a major part of the past decade in creativity assessment, such as changing the task instructions to emphasize quality (Said-Metwaly et al., 2019), having judges provide subjective ratings of the responses (Benedek, Mühlmann, Jauk, \& Neubauer, 2013; Silvia et al., 2008), and using automated methods to evaluate the semantic distance of the responses (Green, 2016; Hass, 2017; Kenett, 2019). 
It is possible that the null effects of caffeine on idea generation reflect the emphasis of the ATTA on how many ideas people can generate, not how good the ideas might be. Future work should explore a wider range of tasks and scoring methods that better separate idea quantity and quality. Along those lines, creativity assessment offers many idea-generation tasks beyond the classic divergent thinking framework. Other tasks, like creating original metaphors in figurative language tasks (Beaty, Silvia, \& Benedek, 2017; Primi, 2014) or creating witty ideas in humor production tasks (Nusbaum, Silvia, \& Beaty, 2017; Ruch \& Heinz, 2019), seem promising for future work.

Interestingly, neither caffeine expectancy, i.e., individual differences in caffeine expectancies on one's behavior, nor believing that one took a caffeine or the placebo capsule were associated with problem solving or with idea generation, with the exception of the link between caffeine expectancy and fewer problems solved with insight. Although previous work shows that caffeine expectancy can influence both behavior and physiology (Kirsch \& Weixel, 1988), our findings suggest that enhance problem solving was the result of the active ingredient in the caffeine capsules. Future studies will need to further examine the relationship between caffeine expectancy and insight solutions.

Interestingly, participants who consumed the caffeine capsule were approximately at chance for guessing if they were in the caffeine or in the placebo condition. Participants who consumed the placebo capsule, however, were more likely to correctly guess that they were in the placebo condition. These results are in line with previous literature showing that discrimination is typically low after consuming lower doses of caffeine (Griffiths et al., 1990).

A between-subject design was used in the present study because it is difficult to find parallel and interchangeable forms of many creativity tasks. Open-ended creativity tasks can also 
have substantial practice effects because participants develop models of the tasks and ideageneration strategies that will carry over from one time to the next (Gilhooly, Fioratou, Anthony, \& Wynn, 2007). Nevertheless, a natural next step for future studies is to use a within-subject design to control for individual differences, ideally with counterbalanced tasks. The present experiment used a fixed task order, but varying the task order could reveal if the effects of caffeine shifted over time. The effects of caffeine have a time course that is difficult to estimate for a sample, given variability in body weight and caffeine tolerance, but a fixed order makes it likely that some tasks will be more strongly affected than others. 


\section{References}

Anderson, K. J., Revelle, W. \& Lynch, M. J. (1989). Caffeine, impulsivity, and memory scanning: A comparison of two explanations for the Yerkes-Dodson Effect. Motivation and Emotion, 13(1), 1-20.

Baas, M., De Dreu, C. K., \& Nijstad, B. A. (2008). A meta-analysis of 25 years of moodcreativity research: Hedonic tone, activation, or regulatory focus? Psychological Bulletin, 134(6), $779-806$.

Baird, B., Smallwood, J., Mrazek, M. D., Kam, J. W., Franklin, M. S., \& Schooler, J. W. (2012). Inspired by distraction: Mind wandering facilitates creative incubation. Psychological Science, 23(10), 1117-1122.

Barry, R. J., Johnstone, S. J., Clarke, A. R., Rushby, J. A., Brown, C. R., \& McKenzie, D. N. (2007). Caffeine effects on ERPs and performance in an auditory Go/NoGo task. Clinical Neurophysiology, 118(12), 2692-2699.

Barry, R. J., Rushby, J. A., Wallace, M. J., Clarke, A. R., Johnstone, S. J., \& Zlojutro, I. (2005). Caffeine effects on resting-state arousal. Clinical Neurophysiology, 116(11), 2693-2700.

Bastia, E., \& Schwarzschild, M. A. (2003). DARPP chocolate: A caffeinated morsel of striatal signaling. Science Signaling, 165, PE2.

Beaty, R. E., Benedek, M., Silvia, P. J., \& Schacter, D. L. (2016). Creative cognition and brain network dynamics. Trends in Cognitive Sciences, 20, 87-95.

Beaty, R. E., Silvia, P. J., \& Benedek, M. (2017). Brain networks underlying figurative language production. Brain and Cognition, 111, 163-170.

Benedek, M. (2018). The neuroscience of creative idea generation. In Exploring Transdisciplinarity in Art and Sciences (pp. 31-48). Springer, Cham. 
Benedek, M., \& Fink, A. (2019). Toward a neurocognitive framework of creative cognition: The role of memory, attention, and cognitive control. Current Opinion in Behavioral Sciences, 27, 116-122.

Benedek, M., Mühlmann, C., Jauk, E., \& Neubauer, A. C. (2013). Assessment of divergent thinking by means of the subjective top-scoring method: Effects of the number of topideas and time-on-task on reliability and validity. Psychology of Aesthetics, Creativity, and the Arts, 7(4), 341-349.

Benedek, M., Panzierer, L., Jauk, E., \& Neubauer, A. C. (2017). Creativity on tap? Effects of alcohol intoxication on creative cognition. Consciousness and Cognition, 56, 128-134.

Bowden, E. M., \& Jung-Beeman, M. (2003). Normative data for 144 compound remote associate problems. Behavior Research Methods, Instruments, \& Computers, 35, 634-639.

Brunyé, T. T., Mahoney, C. R., Lieberman, H. R., \& Taylor, H. A. (2010). Caffeine modulates attention network function. Brain and Cognition, 72(2), 181-188.

Chan, E. Y., \& Maglio, S. J. (2019). Coffee cues elevate arousal and reduce level of construal. Consciousness and Cognition, 70, 57-69.

Chrysikou, E. G. (2019). Creativity in and out of (cognitive) control. Current Opinion in Behavioral Sciences, 27, 94-99.

Dawkins, L., Shahzad, F. Z., Ahmed, S. S., \& Edmonds, C. J. (2011). Expectation of having consumed caffeine can improve performance and mood. Appetite, 57, 597-600.

Fine, B. J., Kobrick, J. L., Lieberman, H. R., Marlowe, B., Riley, R. H., \& Tharion, W. J. (1994). Effects of caffeine or diphenhydramine on visual vigilance. Psychopharmacology, 114(2), 233-238. 
Gilhooly, K. J., Fioratou, E. E., Anthony, S. H., \& Wynn, V. V. (2007). Divergent thinking: Strategies and executive involvement in generating novel uses for familiar objects. British Journal of Psychology, 98, 611-625.

Glade, M. J. (2010). Caffeine—not just a stimulant. Nutrition, 26(10), 932-938.

Goff, K., \& Torrance, E. P. (2002). Abbreviated Torrance Test for Adults manual. Bensenville: Scholastic Testing Services.

Green, A. E. (2016). Creativity, within reason: Semantic distance and dynamic state creativity in relational thinking and reasoning. Current Directions in Psychological Science, 25(1), 28-35.

Griffiths, R. R., Evans, S. M., Heishman, S. J., Preston, K. L., Sannerud, C. A., Wolf, B., \& Woodson, P. P. (1990). Low-dose caffeine discrimination in humans. Journal of Pharmacology and Experimental Therapeutics, 252(3), 970-978.

Hass, R. W. (2017). Tracking the dynamics of divergent thinking via semantic distance: Analytic methods and theoretical implications. Memory \& Cognition, 45(2), 233-244.

Hewlett, P., \& Smith, A. (2007). Effects of repeated doses of caffeine on performance and alertness: New data and secondary analyses. Human Psychopharmacology: Clinical and Experimental, 22(6), 339-350.

Huntley, E. D., \& Juliano, L. M. (2012). Caffeine Expectancy Questionnaire (CaffEQ): Construction, psychometric properties, and associations with caffeine use, caffeine dependence, and other related variables. Psychological Assessment, 24(3), 592 - 607.

Kamimori, G. H., McLellan, T. M., Tate, C. M., Voss, D. M., Niro, P., \& Lieberman, H. R. (2015). Caffeine improves reaction time, vigilance and logical reasoning during extended 
periods with restricted opportunities for sleep. Psychopharmacology, 232(12), 20312042.

Kenett, Y. N. (2019). What can quantitative measures of semantic distance tell us about creativity? Current Opinion in Behavioral Sciences, 27, 11-16.

Kowal, M. A., Hazekamp, A., Colzato, L. S., van Steenbergen, H., van der Wee, N. J., Durieux, J., ... \& Hommel, B. (2015). Cannabis and creativity: Highly potent cannabis impairs divergent thinking in regular cannabis users. Psychopharmacology, 232(6), 1123-1134.

Lee, C. S., Huggins, A. C., \& Therriault, D. J. (2014). A measure of creativity or intelligence? Examining internal and external structure validity evidence of the Remote Associates Test. Psychology of Aesthetics, Creativity, and the Arts, 8(4), 446.

Lorist, M. M., Snel, J., Kok, A., \& Mulder, G. (1996). Acute effects of caffeine on selective attention and visual search processes. Psychophysiology, 33(4), 354-361.

Luft, C. D. B., Zioga, I., Banissy, M. J., \& Bhattacharya, J. (2017). Relaxing learned constraints through cathodal tDCS on the left dorsolateral prefrontal cortex. Scientific Reports, 7(1), 2916.

Mitchell, D. C., Knight, C. A., Hockenberry, J., Teplansky, R., \& Hartman, T. J. (2014). Beverage caffeine intakes in the US. Food and Chemical Toxicology, 63, 136-142.

Miyake, A., Friedman, N. P., Emerson, M. J., Witzki, A. H., Howerter, A., \& Wager, T. D. (2000). The unity and diversity of executive functions and their contributions to complex "frontal lobe" tasks: A latent variable analysis. Cognitive Psychology, 41(1), 49-100.

Nusbaum, E. C., Silvia, P. J., \& Beaty, R. E. (2014). Ready, set, create: What instructing people to "be creative" reveals about the meaning and mechanisms of divergent thinking. Psychology of Aesthetics, Creativity, and the Arts, 8, 423-432. 
Nusbaum, E. C., Silvia, P. J., \& Beaty, R. E. (2017). Ha ha? Assessing individual differences in humor production ability. Psychology of Aesthetics, Creativity, and the Arts, 11(2), 231241.

Ostafin, B. D., \& Kassman, K. T. (2012). Stepping out of history: Mindfulness improves insight problem solving. Consciousness and Cognition, 21(2), 1031-1036.

Palmiero, M., Nakatani, C., Raver, D., Belardinelli, M. O., \& van Leeuwen, C. (2010). Abilities within and across visual and verbal domains: How specific is their influence on creativity?. Creativity Research Journal, 22(4), 369-377.

Perry, S. K. (1999). Writing in flow: Keys to enhanced creativity. Writer's Digest Books.

Primi, R. (2014). Divergent productions of metaphors: Combining many-facet Rasch measurement and cognitive psychology in the assessment of creativity. Psychology of Aesthetics, Creativity, and the Arts, 8, 461-474.

Rao, A., Hu, H., \& Nobre, A. C. (2005). The effects of combined caffeine and glucose drinks on attention in the human brain. Nutritional Neuroscience, 8(3), 141-153.

Reverberi, C., Toraldo, A., D'Agostini, S., \& Skrap, M. (2005). Better without (lateral) frontal cortex? Insight problems solved by frontal patients. Brain, 128(12), 2882-2890.

Ruch, W., \& Heintz, S. (2019). Humor production and creativity: Overview and recommendations. In S. R. Luria, J. Baer, \& J. C. Kaufman (Eds.), Creativity and humor (pp. 1-42). London: Academic Press.

Said-Metwaly, S., Fernández-Castilla, B., Kyndt, E., \& Van den Noortgate, W. (2019). Testing conditions and creative performance: Meta-analyses of the impact of time limits and instructions. Psychology of Aesthetics, Creativity, and the Arts. 
Smeekens, B. A., \& Kane, M. J. (2016). Working memory capacity, mind wandering, and creative cognition: An individual-differences investigation into the benefits of controlled versus spontaneous thought. Psychology of Aesthetics, Creativity, and the Arts, 10(4), 389-415.

Smith, A. P., Clark, R., \& Gallagher, J. (1999). Breakfast cereal and caffeinated coffee: Effects on working memory, attention, mood, and cardiovascular function. Physiology and Behavior, 67, 9-17.

Smith, A., Sutherland, D., \& Christopher, G. (2005). Effects of repeated doses of caffeine on mood and performance of alert and fatigued volunteers. Journal of Psychopharmacology, 19, 620-626.

Stevens Jr, C. E., \& Zabelina, D. L. (2019). Creativity comes in waves: an EEG-focused exploration of the creative brain. Current Opinion in Behavioral Sciences, 27, 154-162.

Thompson-Schill, S. L., Ramscar, M., \& Chrysikou, E. G. (2009). Cognition without control: When a little frontal lobe goes a long way. Current Directions in Psychological Science, 18(5), 259-263.

Torrance, E. P. (2008). Torrance Tests of Creative Thinking: Norms-technical manual, verbal forms $A$ and B. Bensenville, IL: Scholastic Testing Service.

Weinberg, B. A., \& Bealer, B. K. (2004). The world of caffeine: The science and culture of the world's most popular drug. New York: Routledge.

Wiley, J., \& Jarosz, A. F. (2012). Working memory capacity, attentional focus, and problem solving. Current Directions in Psychological Science, 21, 258-262.

Zabelina, D. L., \& Andrews-Hanna, J. R. (2016). Dynamic network interactions supporting internally-oriented cognition. Current Opinion in Neurobiology, 40, 86-93. 